\title{
29 Seltene intestinale Tumoren
}

\author{
Dieter Hahnloser, Fabian Grass
}

\subsection{Kapitelzusammenfassung}

Seltene intestinale Tumoren umfassen gastrointestinale neuroendokrine Tumoren (NET), gastrointestinale Stroma Tumoren (GIST), sowie retrorektale Raumforderungen. Obwohl die heterogenen Krankheitsbilder NET und GIST auch im oberen Gastrointestinaltrakt inklusive Pankreas vorkommen, konzentriert sich dieses Kapitel hauptsächlich auf intestinale und kolorektale Lokalisationen, um eine Übersicht der wichtigsten und typischen Charakteristika, sowie diagnostischen und therapeutischen Maßnahmen dieser seltenen Tumorerkrankungen zu erlangen.

\subsection{Neuroendokrine Tumoren (NET)}

\subsubsection{Definition}

Neuroendokrine Tumoren (NET) sind epitheliale Neoplasien mit vorwiegend neuroendokriner (enterochromaffiner) Differenzierung. Histologisch gut differenzierte NET, historisch Karzinoide aber neuerdings vereinfacht neuroendokrine Tumoren genannt, werden im Magen-Darmtrakt, der Lunge, aber auch seltener in den Nieren oder Ovarien vorgefunden. Auf der anderen Seite finden sich undifferenzierte, hochgradige neuroendokrine Karzinome. Das typische karzinoide Syndrom manifestiert sich bei gleichzeitigem Vorliegen von Lebermetastasen und bezeichnet eine Konstellation von Symptomen, welche durch humorale Faktoren (v. a. gesteigerte SerotoninAusschüttung) hervorgerufen werden.

\subsection{2 Ätiologie}

Relativ wenig ist bekannt bezüglich Risikofaktoren für die Entstehung von NET. Am relevantesten ist eine positive Familienanamnese für alle NET Lokalisationen des Magen-Darmtrakts. Weitere Risikofaktoren sind ein erhöhter Body-Mass-Index und Diabetes. Tabak- und Alkoholkonsum erhöhen das Risiko v. a. bei Pankreas-, Lungenund Dünndarmlokalisation. Eine Assoziation mit dem multiplen endokrinen Neoplasie Typ I (MEN-I) Syndrom ist selten. 


\subsubsection{Pathogenese}

Die meisten Aspekte der komplexen Pathogenese von NET sind weiterhin unbekannt. Die Forschung bemüht sich aktuell hauptsächlich um neue Erkenntnisse der molekularen Mechanismen und versucht Biomarker zur Frühdiagnose und zum Einleiten gezielter therapeutischer Maßnahmen ausfindig zu machen. Im Fokus stehen Somatostatinanaloga, die das Potenzial haben, Symptome der Hormonhypersekretion zu kontrollieren und gleichzeitig die Tumorausbreitung zu verlangsamen.

\subsubsection{Inzidenz}

Die Inzidenz von NET ist steigend, $u$. a. bedingt durch frühere Diagnose. Die amerikanische Surveillance, Epidemiology and End Results (SEER) Datenerfassung beobachtete einen 6,4-fachen Anstieg zwischen 1973 (1,09/100.000) und 2012 (6,98/100.000). Gleichzeitig verbesserten sich aber auch die Überlebensraten (overall survival) zwischen 2009-2012 im Vergleich zu 2000-2004 (HR 0,79, $95 \%$ CI 0,73-0,85), was die Fortschritte in der Therapie unterstreicht. NET können im Alter zwischen 20 und 80 Jahren auftreten, wobei die Inzidenz in Patienten um die 60 Jahre am Höchsten ist.

\subsubsection{Epidemiologie}

Die meisten NET befinden sich im Magen-Darmtrakt (55\%) und im bronchopulmonalen System (30\%). Im Magendarm-Trakt ist die Verteilung folgendermaßen: Dünndarm $28 \%$, Appendix 19\%, Pankreas 16\%, Rektum $15 \%$, Kolon $13 \%$ und Magen 9\%. Weitere epidemiologische Eckdaten sind in Tab. 29.1 zusammengefasst.

Tab. 29.1: Übersicht Epidemiologie gemäß Tumorlokalisation.

\begin{tabular}{lllll}
\hline & Dünndarm & Appendix & Kolon & Rektum \\
\hline Geschlechtspräferenz & $\mathrm{M} \geq \mathrm{F}$ & $\mathrm{M}<\mathrm{F}$ & $\mathrm{M}=\mathrm{F}$ & $\mathrm{M}=\mathrm{F}$ \\
\hline $\begin{array}{l}\text { Alter bei Diagnose } \\
\text { Prädominante Lokalisation }\end{array}$ & $\begin{array}{l}60-65 \mathrm{~J} . \\
\text { Ileum (70 } \%)\end{array}$ & $\begin{array}{l}40-50 \mathrm{~J} . \\
\text { Distales } 1 / 3\end{array}$ & $\begin{array}{l}55-65 \mathrm{~J} . \\
\text { rechtsseitig }\end{array}$ & $\begin{array}{l}\text { 56 J. } \\
\text { oberes/mittleres }\end{array}$ \\
\hline Karzinoides Syndrom & $60-80 \%$ & $<10 \%$ & sehr selten & $3-13 \%$ \\
\hline Multizentrizität & $30 \%$ & $4 \%$ & selten & selten \\
\hline 5-Jahres Überlebensrate & $50-60 \%$ & $70-85 \%$ & $40-50 \%$ & $75-88 \%$ \\
\hline Metastasen bei Diagnose & $30 \%$ & $3-5 \%$ & $30-45 \%$ & $2-8 \%$ \\
\hline
\end{tabular}

M: männlich; F: weiblich; J: Jahre 
Fernmetastasen bei Diagnose sind häufig und ein unabhängiger Prognosefaktor mit einer 5-Jahres Überlebensrate von 13-54 \% gegenüber 75-99\% bei lokalen Befunden. Die Inzidenz einer Peritonealkarzinose beträgt bis zu $17 \%$. Die lokale Rezidivrate beträgt bis zu $35 \%$.

\subsubsection{Klassifikation}

Klassifikation und Nomenklatur von NET sind komplex; dies ist durch organspezifische, uneinheitliche Terminologie und histologische Gradierungen bedingt. So können morphologisch ähnliche NET je nach Ursprungsorgan unterschiedlich bezeichnet werden, und zum jetzigen Zeitpunkt gibt es keine einheitliche Nomenklatur, Gradierung oder Stadieneinteilung. Es gibt aber auch Parallelen, wie z. B. mitotischer Index und lokale Ausbreitung. Die 2 wesentlichen Kategorien für NET des MagenDarmtrakts sind die histogenetisch und molekular unterschiedlichen gut differenzierten NET oder Karzinoide und die neuroendokrinen Karzinome, wobei eine Transformation eines Karzinoids zu einem Karzinom sehr ungewöhnlich ist. Gemeinsam ist allen Tumorzellen die Sekretion von neuroendokrinen Tumormarkern (v. a. Synaptophysin und Chromogranin), wobei diese bei gut differenzierten Läsionen häufiger ist.

Die Klassifikation gemäß histologischen Kriterien fokussiert auf Proliferationsrate für die Gradierung bei Berücksichtigung des Mitoseindex und des sogenannten Ki-67 labeling index, eines weiteren histologischen Proliferationsparameters. Die 2017 WHO Klassifikation (AJCC Cancer Staging Manual, 8th ed.) berücksichtigt zudem eine dreiteilige Gradierung (G1-G3) gemäß Histologie, Tab. 29.2:

Tab. 29.2: Histologische Kriterien zur Gradierung von NET (WHO/AJCC 2017).

\begin{tabular}{ll}
\hline Grad & Definition \\
\hline Gx & unzuteilbar \\
\hline G1 & $<2$ Mitosen $/ 10$ HPF, Ki-67 Index $<3 \%$ \\
\hline G2 & $2-20$ Mitosen $/ 10$ HPF, Ki-67 Index 3-20\% \\
\hline G3 & $>20$ Mitosen $/ 10$ HPF, Ki-67 Index $>20 \%$ \\
\hline
\end{tabular}

HPF - high-power field

\subsubsection{Symptomatik}

NET manifestieren sich klinisch durch:

- Das karzinoide Syndrom (Sekretion von Serotonin oder anderen vasoaktiven Substanzen): häufige Manifestationen sind Erröten (,flushing“, in 90\% der Fälle), 
Diarrhö (80\%), bronchoasthmatische Symptome und die als Hedinger-Syndrom beschriebene Trikuspidal- oder Pulmonalklappeninsuffizienz.

- Chronische/rezidivierende Bauchschmerzen, evtl. Darmverschluss.

- Rechtseitige Oberbauchschmerzen oder Hepatomegalie bei Vorliegen von Lebermetastasen.

- Früheinsetzendes Sättigungsgefühl.

50-70 \% der NET des gastroenteropankreatischen Systems sind bei Erstdiagnose bereits metastasiert. Davon sind jedoch in 20-50\% der Fälle Primärtumoren nicht auffindbar.

\subsubsection{Diagnostik}

NET werden mittels CT oder MRI (u. a. zur Detektion hypervaskulärer Lebermetastasen) und anhand spezialisierter Bildgebungen diagnostiziert:

- Octreoscan (= Indium-111 Pentetreotid), wichtig zur Identifizierung von Fernmetastasen v. a. bei gut differenzierten Läsionen mit vielen Somatostatin Rezeptoren

- Dotatate (= Gallium Ga-68 markierte Somatostatinanaloga) Positron Emission Tomographie (PET) CT, wichtig um die Therapierbarkeit mit Somatostatinanaloga abzuschätzen (Abb. 29.1).

Falls der Primärherd bei metastasierendem NET nicht bekannt ist wird eine diagnostische Gastro- und/oder Kolonoskopie durchgeführt, bei besonderer Beachtung des terminalen Ileums. Videokapseln werden nicht empfohlen, da die Gefahr eines Darmverschlusses bei intestinaler NET Lokalisation besteht.

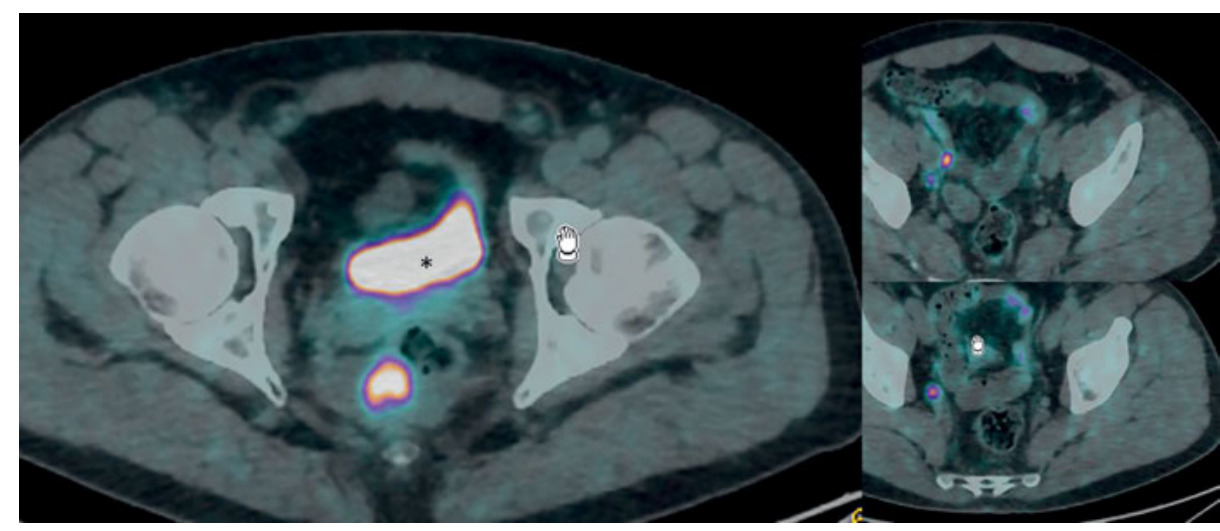

Abb. 29.1: DOTATEC PET-CT eines rektalen NET (G1) (linkes Bild, * = Harnblase) mit rechtsseitigen iliakal internen Lymphknotenmetastasen (rechte Bilder). 
Bei negativer Bildgebung sollte eine chirurgische Exploration erfolgen, wobei NET des Dünndarms nicht selten allein durch ihre bildgebend nachweisbare mesenteriale Lymphadenopathie erkennbar sind.

Immunohistochemische diagnostische Methoden sind komplex und hängen vom jeweiligen Ursprungsorgan ab. Urin sollte auf die Serotonin-Metaboliten 5-HIAA getestet werden, wobei Sensitivität und Spezifität bei gleichzeitigem Vorliegen des karzinoiden Syndroms bei $90 \%$ liegen. Chromogranin A-Serumkonzentration hat weniger als (unspezifischer) Screening-Test aber viel mehr als Tumormarker bei etablierter Diagnose eine wichtige Rolle.

\subsubsection{Differentialdiagnosen}

Das karzinoide Syndrom, welches sich oft durch „flushing“ und unerklärliche, chronische und therapieresistente Diarrhö manifestiert, ist unspezifisch. Die Differentialdiagnose von Diarrhö ist weit gefasst und beinhaltet auch Gastrinome oder VIPome, wobei physiologische Prozesse und Medikamente zum „flushing“ führen können.

\subsubsection{Therapie konservativ}

Die chirurgische Behandlung ist der Goldstandard, um Symptome und Tumorausbreitung vorzubeugen. Besonders bei Patienten mit Hormonhypersekretion ist die Somatostatinanalogatherapie (durch Octreotide oder Lanreotide) von erheblichem Nutzen, verbunden mit wenig Nebeneffekten und Symptomkontrolle in bis zu $88 \%$ der Patienten. Therapie von asymptomatischen, nicht resezierbaren Tumoren muss individualisiert werden, häufig durch Observation bis zur Tumorprogression. Systemische Therapie kann bei Tumorprogression trotz Somatostatinanaloga-Therapie erwogen werden. Die Wirkung ist aber umstritten.

Moderne Therapieoptionen, die aktuell noch erforscht werden, umfassen Immuno- und Molekulartherapien (vascular endothelial growth factor [VEGF, z. B. Everolimus] und mammalian target of rapamycin [mTOR] Inhibitoren).

\subsubsection{Therapie operativ}

Schon bei kleinen Dünndarm-Tumoren von $<10 \mathrm{~mm}$ ist oftmals ein lokal aggressives Verhalten mit Serosapenetration oder lymphatischer Metastasierung zu beobachten. Deshalb darf die Radikalität der Operation nicht von der Tumorgröße beeinflusst werden. Eine prophylaktische Cholezystektomie aufgrund des erhöhten Vorkommens von Gallensteinen unter Somatostatinanaloga-Therapie wird heute nicht mehr empfohlen, da die Steine meistens asymptomatisch sind. Eine darmsparende Resektion 
mit systematischer Lymphadenektomie unter Mitnahme der regionalen Lymphknoten entlang der A. mesenterica superior (mind. 6 resezierte Lymphknoten) ist hingegen wichtiger Bestandteil der operativen Therapie, wobei das Muster des lymphatischen Befalls entscheidend ist (z. B. „skip“-Befall mit Auslassen eines LK-Relais). Aufgrund der Multifokalität (30\%) muss der ganze Dünndarm palpiert werden. Die prophylaktische Resektion des Dünndarmprimarius ist auch im Stadion IV empfohlen.

Bei gastrischer und rektaler Lokalisation wird empfohlen, kleine Läsionen $(<2 \mathrm{~cm})$ ohne histopathologische Risikofaktoren endoskopisch oder lokal-chirurgisch abzutragen, wobei größere Läsionen eine radikale Resektion benötigen.

Die optimale chirurgische Therapie für NET des Appendix ist umstritten. Die meisten Appendix NET werden als „Inzidentalome“ pathologisch entdeckt. Da die Prognose von der Tumorgröße abhängt, wird bei Läsionen von $>2 \mathrm{~cm}$ und/oder Befall des Mesoappendix gemeinhin eine onkologische Rechts-Hemikolektomie empfohlen. Die Grauzone betrifft Läsionen zwischen 1-2 cm, wobei für weniger als $1 \mathrm{~cm}$ eine Appendektomie ausreicht.

NET des Kolons werden oft im fortgeschrittenen Stadium mit lokaler Ausbreitung v. a. rechtsseitig entdeckt (bis $\mathrm{zu} \mathrm{2}_{2} / 3$ mit Nodal- oder Fernmetastasen bei Diagnose) und werden wie Adenokarzinome behandelt, d.h. mittels formeller partieller Kolektomie und regionaler Lymphadenektomie.

Bei Vorliegen von Lebermetastasen ist das chirurgische Vorgehen der Goldstandard, wobei das Ausmaß des Befalls entscheidend ist. Resektion von Lebermetastasen ist indiziert sofern kein G3 Dedifferenzierungsgrad vorliegt, die Resektion sicher durchführbar ist, die Patienten keine durch Rechtsherzklappenfibrose bedingte Rechtsherzinsuffizienz aufweisen und keine nicht behandelbaren Fernmetastasen oder Peritonealkarzinose vorliegen. Bei gut differenzierten Tumoren mit nicht resektablen Lebermetastasen, weniger als 50\% Befall und ohne extrahepatische Manifestationen kann eine Lebertransplantation diskutiert werden.

\subsubsection{Besonderheiten}

NET sind oft mit lokaler oder tumorferner Fibrose assoziiert. So können das HedingerSyndrom (s. oben, bis zu $40 \%$ der Patienten mit karzinoidem Syndrom) oder die mesenterische Desmoplasie bei Dünndarm NET zu beachtlicher Morbidität führen und die Prognose beinträchtigen. Die komplexen Vorgänge, welche zu diesem Phänomen führen, werden aktuell vermehrt erforscht.

Wichtig bei Vorliegen eines NET ist zudem die Nachsorge mit Hilfe der oben aufgeführten Tumormarker (Chromogranin A, 5-HIAA) und durch die lokale (G1 und G2, CT oder MRI) oder systemische Bildgebung (G3, Dotatate PET CT). 


\subsection{Gastrointestinale Stroma Tumoren (GIST)}

\subsubsection{Definition}

Gastrointestinale Stroma Tumoren (GIST) sind von mesenchymalen Zellen (Cajal pacemaker Zellen) ausgehende und typischerweise subepithelial erscheinende Läsionen. Das maligne Potenzial von GIST reicht von gutartigen kleinen Neoplasien zu aggressiven Sarkomen.

\subsection{2 Ätiologie}

Die meisten GIST erfolgen sporadisch ohne etablierte Risikofaktoren, wobei aber auch spezifische Tumorsyndrome auftreten können, z. B. die sogenannte Carney-Triade (GIST - Paragangliom - pulmonäres Chondrom) und die Typ 1 Neurofibromatose (7\% mit GIST).

\subsubsection{Pathogenese}

Entscheidend ist eine Mutation des transmembranösen KIT Rezeptor Tyrosin Kinase Onkogens, welches in bis zu 80\% der GIST vorkommt. In 10\% der Fälle kann eine Mutation des verwandten Tyrosin Kinase Rezeptors PDGFRA vorliegen.

\subsubsection{Inzidenz}

Die Inzidenz bewegt sich im Bereich von 0,7-1,5/100.000 in Europa und den USA, wobei die Inzidenz in asiatischen Ländern vermutlich höher ist. Mehr als 80 \% der GIST kommen in Patienten ab dem 50. Lebensjahr vor.

\subsubsection{Epidemiologie}

GIST machen 20\% der Weichteil-Sarkoma aus. Die meisten GIST befinden sich im Magen (50-60\%), gefolgt von Dünndarm (30-35\%) und Kolon und Rektum (5\%). $40 \%$ der bei Diagnose lokalisierten GIST entwickeln später Metastasen, wobei 10$20 \%$ bei Diagnose bereits metastasiert sind, vorzugsweise zu Leber, Omentum oder Peritoneum. 


\subsubsection{Klassifikation}

Eine eigentlich TNM Klassifikation ist zwar verfügbar (Tab. 29.3), wird aber in der klinischen Praxis kaum angewendet. Wichtiger zur Prognosestellung sind Bildgebung (Tumorheterogenität als Malignitätszeichen), Tumorgröße und -lokalisation (erhöhtes Metastasenrisiko bei Dünndarmlokalisation) und Mitoserate. Lymphknotenbefall ist sehr selten und eine Dissektion deshalb meistens nicht notwendig. Tumorruptur wird als unabhängiger prognostischer Risikofaktor anerkannt, ist aber in der gängigen TNM Klassifikation kein Kriterium.

Tab. 29.3: TNM staging (AJCC 2017).

\begin{tabular}{ll}
\hline & Kriterium \\
\hline Tx & unzuteilbar \\
\hline T1 & keine Tumorevidenz \\
\hline T2 & $\leq 2 \mathrm{~cm}$ \\
\hline T3 & $2-5 \mathrm{~cm}$ \\
\hline T4 & $5-10 \mathrm{~cm}$ \\
\hline N0 & $\geq 10 \mathrm{~cm}$ (größte Dimension) \\
\hline N1 & keine regionalen LK oder unbekannter \\
\hline LK-Status
\end{tabular}

\subsubsection{Symptomatik}

Die meisten GIST sind symptomatisch bei Diagnosestellung; nur ca. $25 \%$ sind Inzidentalome bei Bildgebung und 5\% werden bei der Autopsie diagnostiziert. GIST verursachen typischerweise Blutungen (entweder intraluminal oder -peritoneal), Anämie und Bauchschmerzen. Selten wird eine Notfalloperation aufgrund schwerer Blutung, offener Perforation oder Obstruktion benötigt. 


\subsubsection{Diagnostik}

Für gastrische und rektale Lokalisationen ist der endoskopische Ultraschall hilfreich. Kontrastverstärkte abdomino-pelvine CT oder MRI sind fürs Staging ausreichend, da Metastasen außerhalb des Abdomens nur ausgesprochen selten vorkommen. GIST sollten bei Bedarf endoskopisch (oder ggf. perkutan falls Lebermetastasen) biopsiert werden um eine Tumorausdehnung in die Bauchhöhle zu vermeiden.

\subsubsection{Differentialdiagnosen}

Leiomyome und Leiomyosarkome, maligne Melanome, Schwannome, desmoide und myofibroblastische Tumoren präsentieren sich ähnlich der GIST als subepitheliale Läsionen. Immunohistochemische und molekularanalytische Techniken werden zur Diagnosesicherung beigezogen.

\subsubsection{Therapie konservativ}

Der Goldstandard der GIST Behandlung ist die Chirurgie. Läsionen von < 2 cm können beobachtet oder endoskopisch abgetragen werden.

\subsubsection{Therapie operativ}

\section{Lokalisierte Låsionen}

Entscheidend ist die R0 Resektion bei gleichzeitigem Intakthalten der typischerweise dünnen Pseudokapsel dieser weichen und fragilen Tumoren, wobei makroskopische Margen von 1-2 cm ausreichen (<1\% LK-Metastasen, außer bei pädiatrischen und jungen Patienten < 40 Jahre mit sogenannten pediatric-like GIST [20-59\% positive LK]). Kleine GIST des Magens und Dünndarms können laparoskopisch abgetragen werden. Präoperative Imatinib-Verabreichung zur Tumorverkleinerung kann bei extensiver Chirurgie, z. B. bei Duodenumlokalisation, erwogen werden. Insgesamt wurde ein 5-Jahres-recurrence free survival von 70,5\% beschrieben, wobei der wichtigste prognostische Faktor neben Tumorgröße und -lokalisation die mitotische Rate ist. Bei hohem Rezidivrisiko kann adjuvante Imatinib-Verabreichung diskutiert werden.

\section{Fortgeschrittene Pråsentation}

Bei fortgeschrittenem, metastasierendem GIST kann durch Imatinib der Krankheitsverlauf in über $80 \%$ der Fälle stabilisiert werden, wobei die Therapie bis zur Progression (typischerweise nach 12-36 Monaten) kontinuierlich verabreicht werden muss. 
Die Debulking-Chirurgie bei fortschreitender Krankheit ist v. a. zur Symptombekämpfung indiziert. Radiotherapie kann selektiv zur Palliation beitragen.

\section{Pråvention}

Da keine Risikofaktoren für sporadische GIST bekannt sind, ist die Prävention schwierig. Deshalb fokussieren neue Studien v. a. auf die beste Dosierung und Timing der adjuvanten Imatinib Verabreichung.

\subsection{Retrorektale Raumforderungen}

\subsubsection{Definition}

Der retrorektale oder präsakrale Raum wird anterior, posterior und inferior durch die jeweilige präsakrale, mesorektale und retrosakrale Faszie, lateral durch Ureter und Iliakalgefäße und superior durch die peritoneale Reflektionsline begrenzt. Dieser virtuelle Raum besteht primär aus Bindegewebe (Abb. 29.2). Die äußerst seltenen und heterogenen retrorektalen Tumoren können aufgrund von Malignität oder potentieller maligner Transformation chirurgische Resektion benötigen.

\subsection{2 Ätiologie}

Gewebe diverser embryologischer Abstammung können zu kongenitalen, neurogenen, ossären gemischten Tumoren führen.

\subsubsection{Malignität}

Insgesamt wird mit einem Anteil von ca. 29\% maligner Raumforderungen gerechnet. Das Malignitätsrisiko dürfte bei soliden Raumforderungen mit bis zu 57\% deutlich höher liegen.

\subsubsection{Inzidenz}

Aufgrund der Seltenheit kann keine absolute Inzidenz angegeben werden. Kongenitale, angeborene Raumforderungen sind mit etwa $2 / 3$ der Gesamtzahl am häufigsten. 


\subsubsection{Klassifikation}

Eine Klassifikation in maligne und benigne Läsionen ist am sinnvollsten, wobei die Bildgebung zur Diagnosestellung entscheidend ist. Tab. 29.4 gibt eine Übersicht über die häufigsten präsakralen Tumoren.

Tab. 29.4: Klassifikation präsakraler Tumoren. Übersicht der häufigsten benignen und malignen präsakralen Tumoren.

\begin{tabular}{lll}
\hline & Benigne & Maligne \\
\hline kongenitale Tumoren & $\begin{array}{l}\text { Teratom, Epidermoidzyste, } \\
\text { Dermoidzyste, Rektumduplikatur, } \\
\text { sakrale Meningozele }\end{array}$ & Chordom, Teratolarzinom \\
\hline neurogene Tumoren & Neurofibrom & Neuroblastom, Ependymom, Schwannom \\
\hline ossäre Tumoren & Riesenzelltumor, Osteoblastom & $\begin{array}{l}\text { Osteosarkom, Ewing-Sarkom, Chondro- } \\
\text { sarkom }\end{array}$ \\
\hline $\begin{array}{l}\text { Tumoren } \\
\text { entzündliche }\end{array}$ & Abszess, Fremdkörpergranulom & \\
\hline sonstige Tumoren & $\begin{array}{l}\text { Lipom, Fibrom, Leiomyom, } \\
\text { Desmoid, Hämangiom }\end{array}$ & Liposarkom, Fibrosarkom \\
\hline
\end{tabular}

\subsubsection{Symptomatik}

Symptome (Schmerzen) sind selten; die Raumforderungen sind meistens asymptomatisch bis zur Diagnose im fortgeschrittenen, großen Tumorstadium.

\subsubsection{Diagnostik}

CT und MRI, durch spezialärztliche proktologische, gynäkologische und urologische Untersuchungen zu komplettieren. Der Nutzen von Biopsien ist umstritten und wird aufgrund des tumor seedings und des Infektionsrisikos generell in Frage gestellt. Eine Biopsie sollte nur im Ausnahmefall bei unklarem Befund zur Festlegung des therapeutischen Vorgehens erfolgen. 


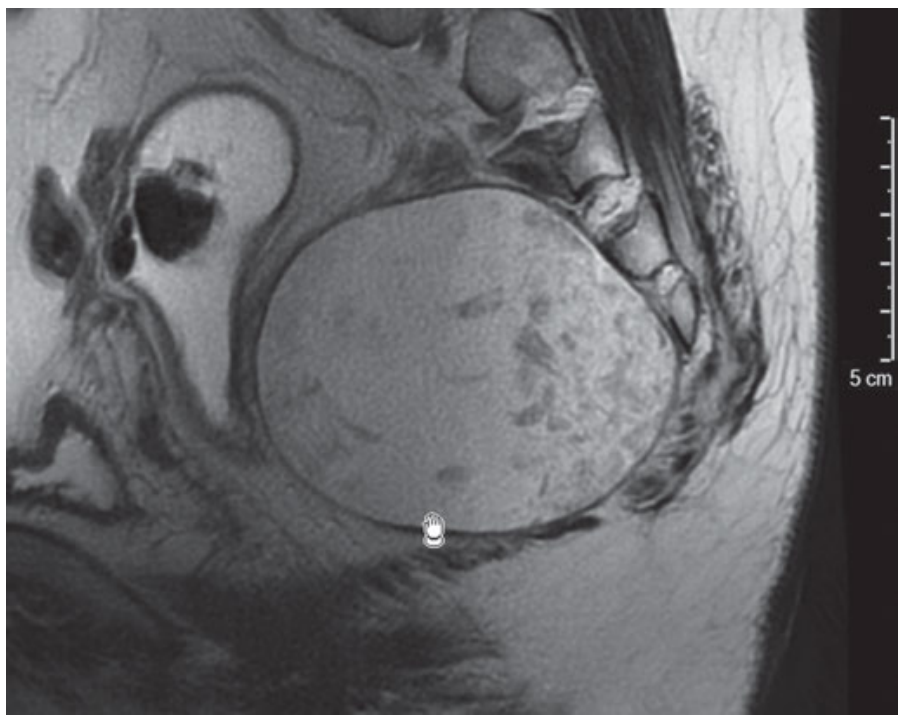

Abb. 29.2: BeckenMRI einer Epidermoidzyste.

\subsubsection{Differentialdiagnosen}

Zur Differentialdiagnose gehören Tumoren der anorektalen und urogenitalen Sphäre mit lymphogener Ausbreitung. Diesbezüglich kann festgehalten werden, dass sekundäre präsakrale Raumforderungen häufiger sind als primäre.

\subsubsection{Therapie operativ}

Die Wahl des chirurgischen Zugangs hängt von der Tumorgröße, -lokalisation undmalignität ab, wobei transabdominale, transperineale, parasakrale oder kombinierte Zugänge möglich sind. Entscheidend für die Wahl des geeigneten Zugangs ist das S3 Sakral-Level. Tumoren unterhalb des dritten Sakralwirbels können durch einen parasakralen Zugang reseziert werden.

\section{Weiterführende Literatur}

Chereau N, Lefevre JH, Meurette G, et al. Surgical resection of retrorectal tumours in adults: longterm results in 47 patients. Colorectal Dis. 2013;15(8):e476-482.

Dasari A, et al. Trends in the Incidence, Prevalence, and Survival Outcomes in Patients with Neuroendocrine Tumors in the United States. JAMA Oncol. 2017;3:1335-1342. doi:10.1001/ jamaoncol.2017.0589.

Graadt van Roggen JF, van Velthuysen ML, Hogendoorn PC. The histopathological differential diagnosis of gastrointestinal stromal tumours. J Clin Pathol. 2001;54(2):96-102. 
Hassan I, Wietfeldt ED. Presacral tumors: diagnosis and management. Clin Colon Rectal Surg. 2009;22(2):84-93.

Hopper L, Eglinton TW, Wakeman C, Dobbs BR, Dixon L, Frizelle FA. Progress in the management of retrorectal tumours. Colorectal Dis. 2016;18(4):410-417.

Joensuu H, Hohenberger P, Corless CL. Gastrointestinal stromal tumour. Lancet. 2013;382(9896):973-983.

Joensuu H, Vehtari A, Riihimaki J, et al. Risk of recurrence of gastrointestinal stromal tumour after surgery: an analysis of pooled population-based cohorts. Lancet Oncol. 2012;13(3):265-274.

Leoncini E, Carioli G, La Vecchia C, Boccia S, Rindi G. Risk factors for neuroendocrine neoplasms: a systematic review and meta-analysis. Ann Oncol. 2016;27:68-81. doi:10.1093/annonc/mdv505.

Maggard MA, O'Connell JB, Ko CY. Updated population-based review of carcinoid tumors. Ann Surg. 2004;240:117-122.

Miettinen M, Lasota J. Gastrointestinal stromal tumors: pathology and prognosis at different sites. Semin Diagn Pathol. 2006;23(2):70-83.

Niederle B, et al. ENETS Consensus Guidelines Update for Neuroendocrine Neoplasms of the Jejunum and Ileum. Neuroendocrinology. 2016;103:125-138. doi:10.1159/000443170.

Rindi G, et al. TNM staging of midgut and hindgut (neuro) endocrine tumors: a consensus proposal including a grading system. Virchows Arch. 2007;451:757-762. doi:10.1007/ s00428-007-0452-1. 
